\title{
Results from the Fréjus experiment on nucleon decay modes with charged leptons
}

Fréjus Collaboration

\author{
Ch. Berger, M. Fröhlich, H. Mönch, R. Nisius, F. Raupach, P. Schleper \\ I. Physikalisches Institut der RWTH Aachen ${ }^{1}$, W-5100 Aachen, Federal Republic of Germany
}

Y. Benadjal, D. Blum, C. Bourdarios, B. Dudelzak, P. Eschstruth, S. Jullian, D. Lalanne, F. Laplanche, C. Longuemare ${ }^{2}$, C. Paulot ${ }^{3}$, O. Perdereau, Ph.Roy, G. Szklarz

Laboratoire de l'Accélérateur Linéaire, Centre d'Orsay, Bâtiment 200, F-91405 Orsay, France

L. Behr, B. Degrange, U. Nguyen-Khac, S. Tisserant

LPNHE-Ecole Polytechnique, Route de Saclay, F-91128 Palaiseau, France

C. Arpesella ${ }^{4}$, P. Bareyre, R. Barloutaud, A. Borg, G. Chardin, J. Ernwein, J.F. Glicenstein, L. Mosca, L. Moscoso

DPhPE, CEN-Saclay, F-91191 Gif-sur-Yvette Cedex, France

J. Becker ${ }^{5}$, K.H. Becker, H.J. Daum, B. Jacobi, B. Kuznik, J. Löffler, H. Meyer, R. Möller, M. Schubnel1 ${ }^{6}$, Y. Wei, P. Wintgen ${ }^{7}$

Bergische Universität-Gesamthochschule Wuppertal ${ }^{8}$, W-5600 Wuppertal, Federal Republic of Germany

Received 8 November 1990

\begin{abstract}
Lower limits on the nucleon lifetime in channels containing at least one positron or muon are given. The analysis is based on 4 years of data taking with the Fréjus detector. Various approaches to determine the background from atmospheric neutrinos are discussed and two different nuclear models are used in event simulations. The limits obtained range from $10^{31}$ years for $p \rightarrow e^{+} K^{* 0}$ to $1.5 \cdot 10^{32}$ years for $p \rightarrow \mu^{+} \gamma$.
\end{abstract}

\section{Introduction}

The ideas of grand unification [1] have stimulated the experimental search for nucleon decay. Results on lower limits of the nucleon lifetime have been obtained using

\footnotetext{
Supported by the BMFT, FRG, under contract number 55AC14P

2 Now at L.P.C. Université de Caen, F-14021 Caen, France

3 Now at Laboratoire GANIL, F-14021 Caen, France

4 Now at Istituto di Fisica, Universitá di Milano, I-20133 Milano, Italy

5 Now at Fakultät für Physik, Universität Freiburg, FRG

6 Now at University of Michigan, Ann Arbor, Michigan USA 48109-1120

7 Now at Fisher Controls GmbH, Solingen, FRG

${ }^{8}$ Supported by the BMFT, FRG, under contract number 55WT84P
}

water Črenkov and iron calorimeter detectors [2-6]. In this paper we present data recorded with the Frejus detector, a fine grain tracking calorimeter allowing for a good pattern recognition of multi-prong events. Here we consider nucleon decay into modes containing at least one charged lepton. Other baryon-number violating processes like nucleon decay into channels with an antineutrino [7] or the neutron-antineutron oscillations [8] have already been published.

The paper is organized as follows: after a short description of the detector and the processing of the data (Sect. 2) the Monte Carlo simulation of the nucleon decay inside the iron nucleus is discussed (Sect. 3). Two different nuclear models have been studied and compared to experimental data.

A careful study of the background from atmospheric neutrinos is mandatory for any nucleon decay analysis. We determine this background by a reanalysis [9] of the Aachen-Padova spark chamber experiment at the CERN proton synchrotron PS (Sect. 4.1) and independently by a Monte Carlo simulation of neutrino interactions in the Fréjus detector [10] (Sect. 4.2). Both methods describe the Fréjus data quite well.

Starting from three different approaches (Sect. 5), we give lifetime limits for 29 decay channels (Sect. 6) and an estimate of the systematic errors (Sect. 7). 


\section{The experiment}

\subsection{The detector}

The Fréjus nucleon decay detector was installed in an underground laboratory located in the middle of the Fréjus alpine road tunnel connecting Modane (France) to Bardonecchia (Italy). The average rock overburden amounts to $1780 \mathrm{~m}$. The $900 \mathrm{t}$ detector is $6 \mathrm{~m}$ in height with an horizontal surface of $6 \mathrm{~m} \times 12.3 \mathrm{~m}$. It is a very fine grain tracking calorimeter with $(5 \mathrm{~mm} \times 5 \mathrm{~mm})$ cells and a calorimetric sampling of $3 \mathrm{~mm}$ of iron plates. 912 vertical planes of flash chambers and 113 planes of Geiger tubes with alternately horizontal and vertical cells provide two orthogonal views. The trigger obtained from the Geiger tubes requires, in a time interval of $320 \mathrm{~ns}$, at least 5 hits in 5 adjacent planes, each plane contributing at most 3 hits. For the nucleon decay modes studied in this paper this results in trigger efficiencies ranging from $48 \%$ for $p \rightarrow \mu^{+} \rho^{0}$ to $98 \%$ for $p \rightarrow e^{+} e^{+} e^{-}$. The efficiency of the detector was constantly monitored by analyzing the muons crossing the apparatus. A detailed description of the detector can be found elsewhere [11].

\subsection{Data taking}

Data taking started in February 1984 with a mass of $240 \mathrm{t}$. The full detector was completed in June 1985. This analysis uses all data until the final shut down of the Frejus detector in September 1988 and represents a total accumulated sensitivity of 2.5 kiloton-years $(\mathrm{kt} \cdot \mathrm{y})$. During this time 1200000 triggers have been recorded, mainly due to almost equal contributions of atmospheric muons and local radioactivity or electronic noise. The contained neutrino events or nucleon decay candidates were identified by visual scanning on a graphic terminal and, independently, using two different pattern recognition programs.

\subsection{Data selection and processing}

Two different particle signatures can be distinguished in our apparatus: showers produced by electrons or photons and tracks produced by muons or charged hadrons. Assigning a vertex to an event and the classification of the prongs originating from it (i.e. the number of tracks and showers) determines the topology of the event. In general, due to the good spatial resolution, showers and tracks are straightforward to identify. This can be seen from the two examples given in Fig. 1. The sensitivity of the results to the detailed scanning criteria, to the procedure in the case of ambiguities (e.g. multiple possibilities for the vertex position) and to the technical treatment (manual or automatic) justifies the various analyses presented in Sect. 5.

All analyses are based on contained events only. An event is contained, if no prong (extended by a safety region taking into account the conversion length of photons, the detector geometry and the cell efficiency) is leaving the detector. The effective detector mass available for nucleon decay vertices is thus reduced. Three different definitions have been used to determine the fiducial mass.

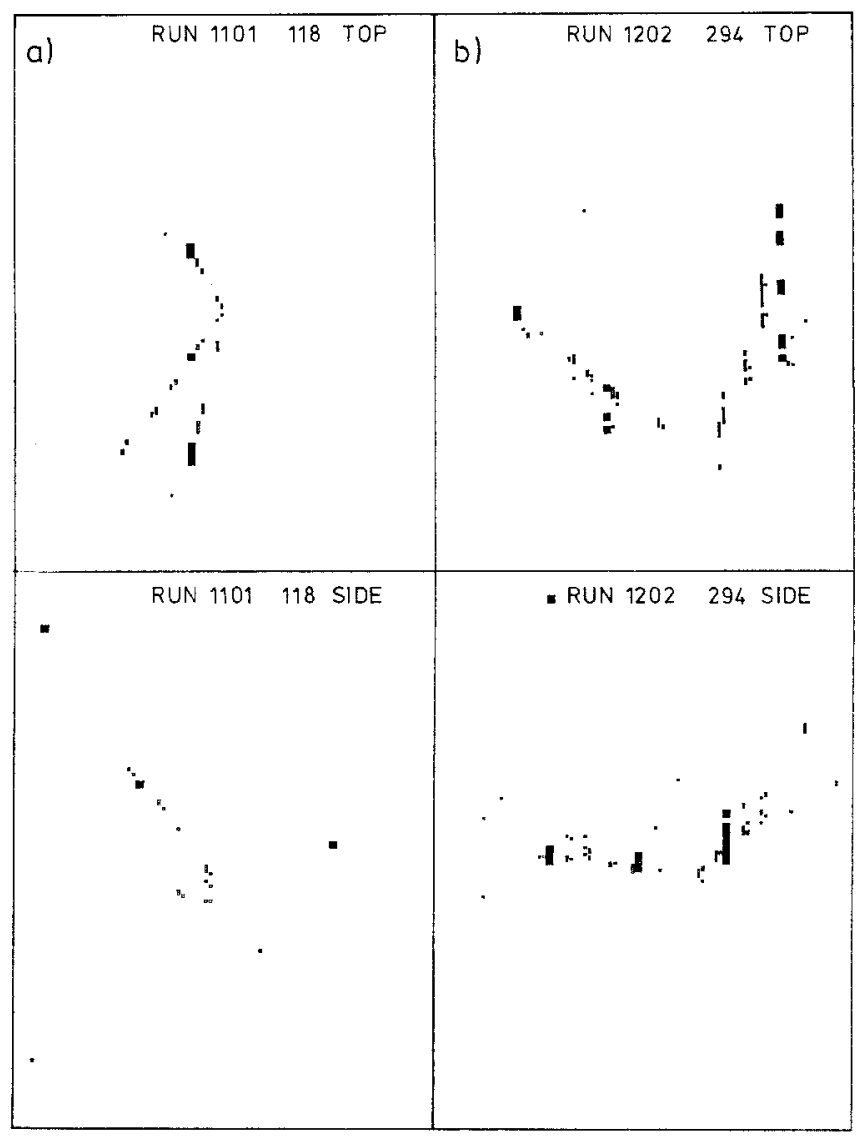

Fig. 1a, b. Fréjus events produced by neutrino interactions. Two orthogonal views are shown. The large squares represent the fired Geiger tubes and the small ones the flash tubes. a a three track event with total energy $E=0.87 \mathrm{GeV}$ and total momentum $p=0.45 \mathrm{GeV}$; b a two shower event with total energy $E=1.18 \mathrm{GeV}$ and total momentum $p=0.96 \mathrm{GeV}$

The number of contained events found inside the fiducial volume ranges from 142 to 153 , depending on the containment criteria. The events with at least two prongs ( $60 \%$ respectively $45 \%$ with visible energies below $2 \mathrm{GeV}$ ) are used for further analyses.

The energy of electromagnetic showers is calculated according to the calibration with a test-detector of identical structure exposed to an electron beam at DESY and to neutral pions produced in charge exchange reactions in a pion beam at Bonn [11]. At energies relevant for nucleon decay the detector response is nearly linear with $7 \mathrm{MeV}$ per flash chamber hit and an energy resolution of $\frac{\Delta E}{E} \cong 15 \%$ at $400 \mathrm{MeV}$.

The energy of non showering particles is obtained from their range by the Bethe-Bloch relation. This leads to an accuracy of $10 \mathrm{MeV}$ for muons with a momentum of $300 \mathrm{MeV}$ perpendicular to the iron plates.

Due to nuclear interactions in the detector only $45 \%$ of the pions with a momentum of $300 \mathrm{MeV}$ reach their nominal range. Therefore a part of the pionic energy is not visible in the detector (e.g. on average $77 \%$ of the total energy is visible for pions with $p=300 \mathrm{MeV}$ ).

Figure 2 shows the distribution of the visible momentum $p=\left|\sum_{\text {all particles }} p_{i}\right|$ vs. the visible energy $E=\sum E_{i}$, 


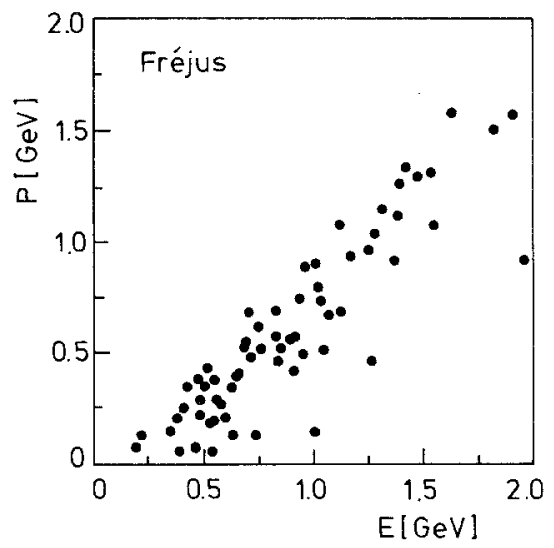

Fig. 2. Measured momentum $p$ vs. energy $E$ for contained Fréjus events with at least 2 prongs (analysis 3 , see Sect. 5.3). If there is more than one possible interpretation of an event the lowest $p / E$ is taken

with $E_{i}^{2}=p_{i}^{2}+m_{i}^{2}$. For this diagram all non showering prongs are considered to be pions. No obvious signal is present in the nucleon decay region $\left(E \approx m_{p}=938 \mathrm{MeV}\right.$, $p \approx 0$ ).

\section{Nuclear effects}

The mesons produced in decays of nucleons bound in nuclei undergo strong interactions with the nuclear matter.

The interaction probabilities for $\eta(\omega)$ mesons are derived from the $\pi N=\eta(\omega) N$ nucleon cross sections using the method of detailed balance [12]. Due to its short decay length the interaction of the $\rho$ is not considered. The probability for $K^{0}$ and $K^{+}$mesons to interact inside the nucleus is negligible $(<5 \%)$.

The interaction of pions produced in nucleon decay is dominated by the production of $\Delta$-resonances smeared out by the Fermi-motion of the nucleons. The models outlined below describe the interaction of pions via the formation of a resonance in the nuclear matter using the experimental pion nucleon scattering cross sections [13]. This resonance, propagating through the nucleus, may decay, if allowed by the Pauli principle, leading to a quasi free pion nucleon scattering or charge exchange reactions. Alternatively the resonance may interact with additional nucleons which may finally lead to the absorption of pions inside the nucleus.

Two models have been developed in order to evaluate the behaviour of pions inside the nucleus. The first (model A) is based on simple hypotheses, while the second (model B) assumes a more detailed knowledge of the nuclear processes.

\subsection{Model A}

In this model the iron nucleus is assumed to be a Fermi gas of noninteracting nucleons. The nuclear density is taken constant and the mean square radius is obtained from electron-nucleus scattering data [14].
In order to take into account the absorption the $\Delta$ scattering amplitude is modified by introducing an absorptive width [15]. This width is determined empirically by a fit to the experimental $\pi$-Fe absorption, inelastic and charge exchange yields.

\subsection{Model B}

This model uses a nuclear density distribution of WoodsSaxon type with nuclear radii obtained from electron nucleus scattering data [14]. The nucleon decay is allowed to take place in different nuclei according to the composition of the detector material ( $87 \%$ of iron). The motion of the nucleons inside the nuclei is described by the local density approach of a Fermi gas model with a maximum Fermi energy of $37 \mathrm{MeV}$ leading to the proper momentum distribution for the different nuclei.

The interaction of the pion-nucleon resonances proceeds via virtual pion exchange exciting $\Delta$-resonances. Concurrently to quasifree pion nucleon scattering the $\Delta$ resonance may interact with additional nucleons either via pion scattering on two nucleons or initiating the process of pion absorption. Since the momentum transfer is degraded in each $\Delta$-resonance formation this model allows for at most three nucleons participating in the genuine pion absorption [16].

The probabilities for the different pion scattering and absorption processes are obtained from isospin considerations, the $\pi N \Delta-, \pi N N$ - and $\pi \Delta \Delta$-couplings taken from [17] and the Breit-Wigner shape of the excited $\Delta$ resonances. Since at least two nucleons participate in pion absorption the probability of this process varies roughly like the nuclear density squared while the scattering probability is proportional to the density. Therefore pion absorption dominates at small radii while scattering takes place at the periphery of the nucleus. Due to this difference the pion absorption is expected to be somewhat smaller compared to the constant density model.

The secondary particles produced in the pion interaction with nuclear matter may interact subsequently with the nuclear fragment initiating an intranuclear cascade. Since protons with energies larger than $100 \mathrm{MeV}$ are visible in the Fréjus detector* the nucleon-nucleus interactions are included in a similar way. The nucleon cross section in nuclear matter is obtained from nucleon-nucleon cross sections [18] using its isospin decomposition [19] and accounting for the two particle correlations of the Fermi gas model. In agreement with observation [20], this ansatz leads to a larger mean free path of nucleons in nuclear matter compared to the naive expectation from nucleon-nucleon scattering data.

\subsection{Comparison of the two models}

Both approaches allow to reproduce roughly the experimental cross sections [21] for the various processes shown in Fig. 3. The agreement between the simulation and the data is sufficient for our purpose especially if one considers the systematic uncertainties in the determination of the measured cross sections. In Sect. 7 we estimate the

* However in the following analyses all tracks are interpreted as pions or muons 

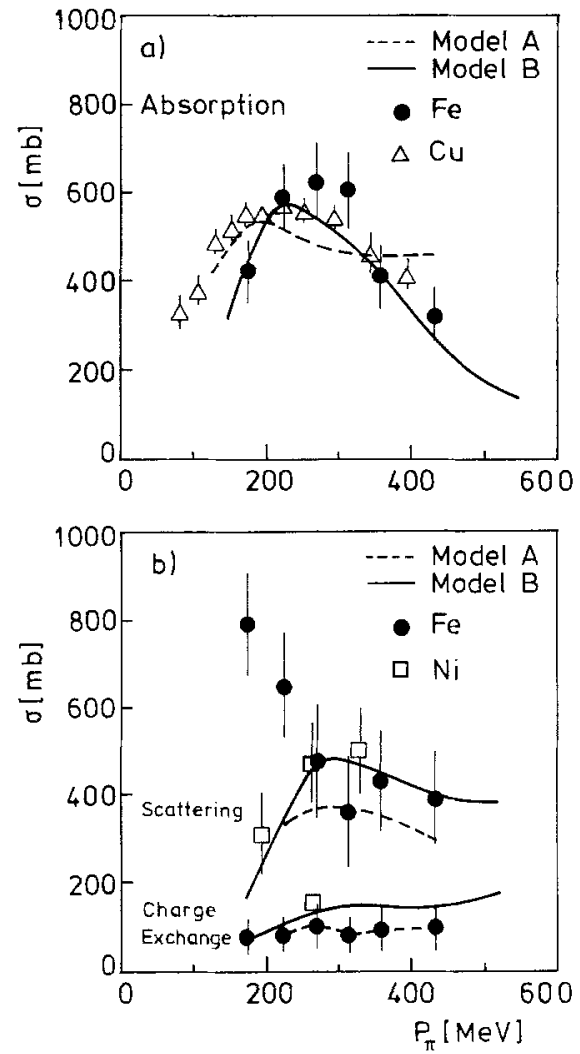

Fig. 3a, b. Comparison of experimental data on $\mathrm{Fe}, \mathrm{Ni}$ and $\mathrm{Cu}[21]$ with the energy dependent cross sections of positively charged pions on iron simulated with the two nuclear models for the various processes. The error bars for $\mathrm{Cu}$ account only for the statistical errors, the systematic errors are however considerably larger. Note that the measurement of the scattering cross section on iron is affected with large systematic uncertainties for momenta $p_{n} \leqq 250 \mathrm{MeV}$

Table 1. The effect of pion nucleus interaction for pions produced in the nucleon decay mode $p \rightarrow e^{+} \pi^{0}$. The numbers indicate the probability of each process

\begin{tabular}{llllll}
\hline Model & $\begin{array}{l}\text { No } \\
\text { interaction }\end{array}$ & $\begin{array}{l}\text { Scat- } \\
\text { tering }\end{array}$ & $\begin{array}{l}\text { Charge } \\
\text { exchange }\end{array}$ & $\begin{array}{l}\text { Absorp- } \\
\text { tion }\end{array}$ & $\begin{array}{l}\pi \text {-pro- } \\
\text { duction }\end{array}$ \\
\hline A & 0.34 & 0.16 & 0.14 & 0.36 & - \\
B & 0.48 & 0.15 & 0.12 & 0.22 & 0.03 \\
\hline
\end{tabular}

influence on the nucleon lifetime limits comparing the results obtained with both models.

As an example Table 1 gives the results of the two models on the influence of the iron nucleus for the decay mode $p \rightarrow e^{+} \pi^{0}$. The pion survival probability is significantly higher in model B (48\%) compared to the simpler model A (34\%). This is mostly due to the different treatment of pion absorption. These differences will reflect as systematic uncertainties in our final results.

\section{Background evaluation}

The contained events in our detector are due to neutrino interactions. The neutrinos are produced in the atmosphere of the earth in hadronic showers induced by primary cosmic rays. Several authors [22-24] have calcu- lated the expected neutrino flux in particular at the location of the Fréjus detector.

The background induced by muons crossing the detector parallel to the plates (and therefore invisible) but with visible secondary hadrons or bremsstrahlung and the background from neutral particles other than neutrinos has been estimated to be less than 3\% [10] and is therefore negligible.

The neutrino background expected in the Fréjus detector has been studied by an experimental method (Sect. 4.1) and by a Monte Carlo simulation (Sect. 4.2).

\subsection{Analysis of the Aachen Padova data*}

The Aachen Padova (AcPd) spark chamber detector [25] was designed for an investigation of $\left(\frac{(-)}{v}\right.$-neutral current reactions in the $\mathrm{GeV}$ energy-region at the CERN-PS. Its sampling structure is very close to that of the Fréjus detector and therefore the shower development and the interaction probability of pions are similar in both detectors.

In transferring these results to the Fréjus experiment we correct for some differences by applying appropriate weight factors:

- The fraction of electron neutrinos is practically negligible in an accelerator beam, whereas it is roughly $50 \%$ for atmospheric neutrinos [22]. In order to correct for this different composition, charged current reactions in the Aachen Padova experiment are treated by replacing with the appropriate frequency the outgoing muon by an electron with the same momentum.

- We correct for the different shape of the atmospheric neutrino flux spectrum compared to that of the CERN PS beam by applying energy dependent weight factors to the Aachen Padova events.

- In order to take into account the Fréjus trigger efficiency an energy dependent correction weight for the Aachen Padova events has been calculated by simulating the response of our detector to the Aachen Padova events. - The lateral size of the Aachen Padova detector is $2 \mathrm{~m} \times 2 \mathrm{~m}$. This results in an acceptance for contained events which depends strongly on their spatial extension in the apparatus. We correct for this effect by applying a geometrical weight to each event.

Neutrino events contributing to the background for nucleon decay should be rather balanced in visible momentum. It has been checked that no correction is necessary to account for the approximately isotropic atmospheric neutrino flux as compared to the neutrino beam perpendicular to the plates.

As we have to process the Aachen Padova events in the same way as the Frejus events we may interpret the point of a strong reinteraction of one of the secondary particles as the vertex of the event. Comparing with the well defined situation in the Aachen Padova $(\vec{v}$-beam experiment we estimate the contribution of these reinteraction vertices to the background for nucleon decay channels to be about $50 \%$.

\footnotetext{
* A detailed description of this analysis can be found in [9]
} 
The data sample of our reanalysis of the Aachen $\mathrm{Pa}$ dova events is equivalent to an exposure of $60 \mathrm{kt} \cdot \mathrm{y}$ for the Fréjus detector to atmospheric neutrinos in the $1 \mathrm{GeV}$ energy region. Using all weighting factors we calculate the expected number of background events for each individual topology of interest in the nucleon decay analysis.

\subsection{Neutrino Monte Carlo simulation}

Using the neutrino flux at the Fréjus underground laboratory [22] we simulate the products of an interaction with a nucleus in the detector by a program which takes into account the neutrino cross sections in the neutrino energy range $\left(0.2 \leqq E_{v} \leqq 20 \mathrm{GeV}\right)$. The nuclear interactions of the produced hadrons are treated with the model B described in Sect. 3.2 while the detector response is simulated with the detector Monte Carlo programs described in Sect. 5.

Dominant processes of the neutrino reactions are quasi-elastic scattering, pion and multi pion production while strange particle production is known to be small and therefore neglected. The results of the simulation are in close agreement with the observed neutrino events [10].

The simulated neutrino events are subject to exactly the same analysis chain as the Fréjus data and the simulated nucleon decay events.

\subsection{Comparison with the data}

Both methods describe our data quite well for $E<2 \mathrm{GeV}$ and topology $\geqq 2$ prongs. Since no global excess of events in the nucleon decay region is observed (see Fig. 2) detailed mode dependent analyses are needed to search for nucleon decay candidates and to set lifetime limits on various decay modes.

Reasonable agreement between the absolute predictions and the data has been found as shown in Fig. 4a$c$, where the multiplicity, the energy and the momentum distributions are presented. The total number of predicted events is 78 in the neutrino Monte Carlo and 55 in the Aachen-Padova data, whereas in the Fréjus experiment 66 events are seen. The systematic error of both predictions amounts to $30 \%$ mainly due to the uncertainties on the neutrino flux and on the nuclear effects.

In a few cases the pattern of a single shower may mimic a multi shower event in the Fréjus detector. The number of events with at least two showers found in the Frejus data ranges from 15 to 24 events depending on the analysis and from 20 to 24 events in the Monte Carlo simulation. The somewhat lower prediction from the Aachen Padova data (about 7 events) may be due to the artificial origin of the $v_{e}$-events.

We account for these uncertainties in the analyses described in Sect. 5 by using different methods of background evaluation.

\section{Nucleon decay analyses}

The naive expectation for the nucleon decay $\sum E_{i}$ $=938 \mathrm{MeV}$ and $\sum p_{i}=0$ is modified by the nuclear effects
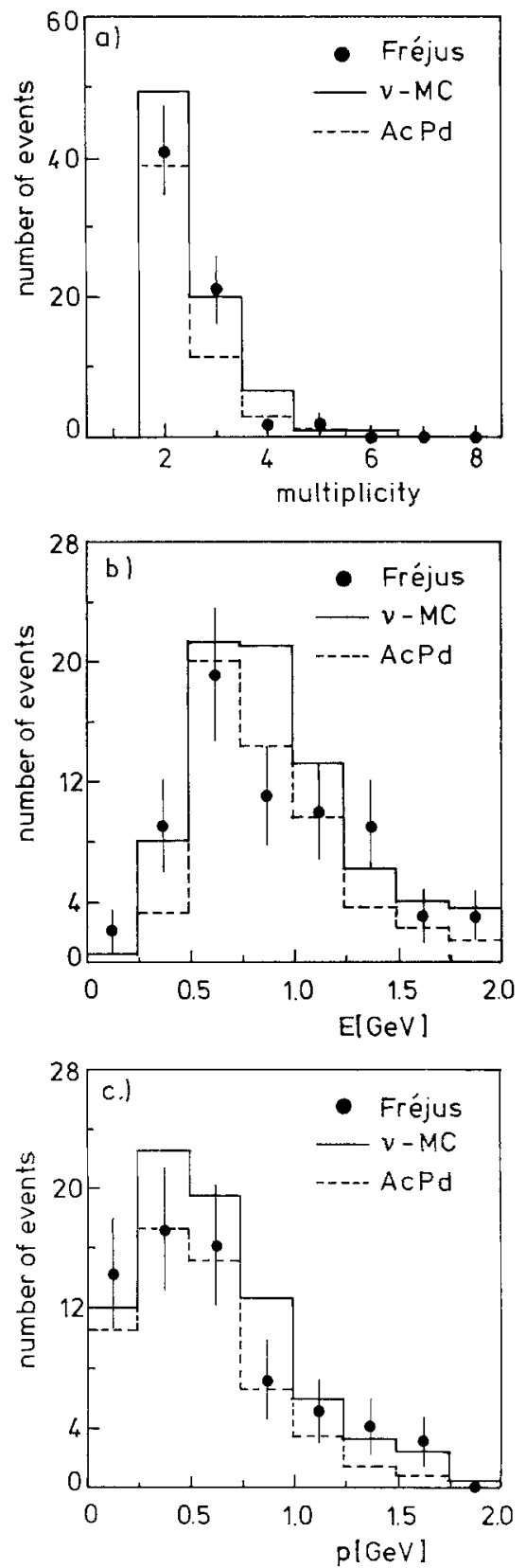

Fig. 4a-c. Comparison of absolute background predictions and the Fréjus data for a multiplicity, $\mathbf{b}$ visible energy and $\mathbf{c}$ visible momentum distributions. The events are classified according to the criteria mentioned in Fig. 2 and taken from analysis 3 (Sect. 5.3)

and the measurement errors. Since there is no unique way to define candidate events their treatment is different in the three analyses presented in this section. This allows us to study the influence of the selection criteria on the results.

The calculation of lower limits on the nucleon lifetime is based on the number of observed candidates, the expected background and the detection efficiency for a given decay mode. These are derived from three sets of data: the contained Fréjus events, the $v$-background and the 250-300 simulated events for a given nucleon decay mode, subject to the same analysis chain inside the framework of each analysis. 
It is a general feature of the three analyses that there is more than one interpretation for $10-20 \%$ of the events. Most of the ambiguities are due to the fact that either a secondary interaction or substructure inside a single shower indicates a second possible vertex. We keep all hypotheses during the analysis and select at the end the most probable for nucleon decay.

For each decay channel, a topological selection and kinematical cuts are applied on both simulated decay and neutrino background events. To be independent from the Fréjus data we define the cut(s) by maximizing the expected lifetime limit with respect to the recognition efficiency and the predicted background.

The three analyses presented below are described in detail in [26].

\subsection{Analysis 1}

5.1.1 Simulation and background. The nucleon decay inside the iron nucleus is treated according to model A. The detector response to the decay particles is simulated by the Monte Carlo program GEANT [27].

The background for nucleon decay is evaluated using the Aachen-Padova data.

5.1.2 Measurement of the events. As for the Fréjus data the simulated nucleon decay events are scanned and measured on a graphic terminal. To guarantee a minimal scanning bias the nucleon decay events are mixed among the various decay modes. A vertex is defined and the hits are associated with tracks or showers in both views using criteria similar to those adopted in the Aachen-Padova analysis [9]. For each event the topology with the best momentum balance is chosen.

A fiducial cut is applied on vertices located less than $50 \mathrm{~cm}$ from the edges of the detector. This leads to an effective mass of $554 \mathrm{t}$ corresponding to a total sensitivity of $1.56 \mathrm{kt} \cdot \mathrm{y}$.

5.1.3 Data analysis. Topological and kinematical cuts (on $E, p$ and meson invariant mass(es)) are used to reject the background. Since no a priori hypothesis of a decay channel is assumed at the scanning level, the accepted topological configurations of scanned events may differ from the expected decay products (cf. Table 2).

Table 2. Results of the three analyses for two decay modes. $S=$ shower, $T=$ Track and $X=$ shower or track. $\varepsilon_{\text {i }}$ denotes the efficiency and $b_{i}$ is the branching ratio into the investigated meson decay modes. $N_{B}$ and $N_{C}$ are the numbers of background and candidate events and the background subtracted limits on $\tau_{N}$ are given
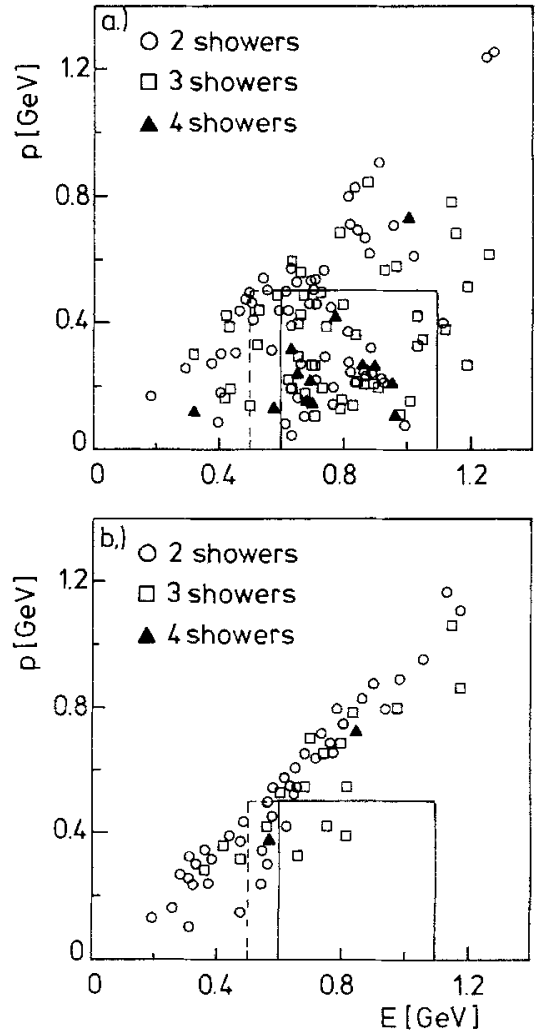

Fig. 5a, b. Scatter plot $p$ vs. $E$ of events with 2,3 or 4 showers for: a Simulated and reconstructed $p \rightarrow e^{+} \pi^{0}$ events b Neutrino interactions from the Aachen-Padova experiment corresponding to a sensitivity of $60 \mathrm{kt} \cdot \mathrm{y}$. The boxes represent the kinematical cuts used to select the nucleon decay candidates. The solid line is for 2 and 3 showers and the dotted line for 4 showers

As an example Fig. 5 shows the $p$ vs. $E$ distributions of simulated $p \rightarrow e^{+} \pi^{0}$ nucleon decay events and Aachen Padova neutrino background. The kinematical cuts to select the candidate events are also presented. Among the 8 Fréjus events belonging to the selected topological configurations $(2,3,4$ showers) none remains inside the chosen cuts.

\subsection{Analysis 2}

5.2.1 Simulation and background. The mesons produced in nucleon decay inside the nucleus are treated according

with $90 \%$ confidence level. $N_{B} \leqq 0.1$ means that no background event has been found but depending on the underlying statistics this corresponds to a $66 \%$ confidence level limit on the background. For the $K_{s}^{0}$ the decay modes $K_{s}^{0} \rightarrow \pi^{+} \pi^{-}$and $K_{s}^{0} \rightarrow \pi^{0} \pi^{0}$ are studied

\begin{tabular}{|c|c|c|c|c|c|c|c|}
\hline Channel & Analysis & Topology & $\sum_{i} \varepsilon_{i} \cdot b_{i}^{\mathrm{a}}$ & $N_{B}^{\mathrm{a}}$ & $N_{C}$ & Sens. $(\mathrm{kt} \cdot \mathrm{y})$ & $\frac{\tau_{N}}{\operatorname{BR}\left(10^{31} \mathrm{y}\right)}$ \\
\hline$p \rightarrow e^{+} \pi^{0}$ & $\begin{array}{l}1 \\
2 \\
3\end{array}$ & $\begin{array}{l}2,3,4 S \\
2 S \text { or } 1 X+2,3 S \\
2,3 S\end{array}$ & $\begin{array}{l}0.23 \\
0.33 \\
0.36\end{array}$ & $\begin{array}{r}0.1 \\
\leqq \\
0.1 \\
0.5\end{array}$ & $\begin{array}{l}0 \\
0 \\
0\end{array}$ & $\begin{array}{l}1.56 \\
2.00 \\
1.58\end{array}$ & $\begin{array}{l}4.4 \\
8.1 \\
7.0\end{array}$ \\
\hline$p \rightarrow \mu^{+} K_{S}^{0}$ & $\begin{array}{l}1 \\
2 \\
3\end{array}$ & $\begin{array}{l}3 T \text { or } 1 T+2,3,4 S \\
2 T \text { or } 2 T+1 X \text { or } \\
\quad 1 T+2 S \text { or } 1 T+2 S+1,2 X \\
3 T \text { or } 1 T+2,3,4 S\end{array}$ & $\begin{array}{l}0.34 \\
0.40\end{array}$ & $\begin{array}{l}1.2 \\
2.2\end{array}$ & $\begin{array}{l}0 \\
1\end{array}$ & $\begin{array}{l}1.56 \\
2.00\end{array}$ & $\begin{array}{l}6.4 \\
5.8\end{array}$ \\
\hline
\end{tabular}

a The various efficiencies and numbers of background events reflect the influence of the different nuclear models and of the chosen cuts 
to model B. A Monte Carlo program especially tuned for low energy hadrons and for the development of electromagnetic showers in a fine grained calorimeter is used to simulate the detector response to particles produced in nucleon decay.

The background for nucleon decay is determined using the neutrino Monte Carlo described in Sect. 4.2. We have simulated neutrino interactions corresponding to a sensitivity of $12.5 \mathrm{kt} \cdot \mathrm{y}$. The same Monte Carlo programs as described above are used to simulate the hadron reinteraction in nuclei and the detector response.

In order to minimize the scanning bias for the vertex determination these neutrino events were mixed with the simulated nucleon decay events and processed together imposing the nucleon decay hypotheses to all events.

5.2.2 Measurement of the events. This analysis uses a pattern recognition program to reconstruct the Fréjus data and the simulated events. Only the vertices are determined visually on a graphic terminal while track finding, association of tracks in the two independent views, particle type identification (showers or tracks) and momentum determination are done by program. The type of each prong is determined using a maximum likelihood test on the transverse profile and on the distribution of hits per plane for hits associated with the prong. These tests yield an identification probability of $85 \%$ for showers and $90 \%$ for non showering particles at $200 \mathrm{MeV}$ and more than $95 \%$ above $400 \mathrm{MeV}$ for both particle types. For a few percent of the events the correlation of prongs in the two independent views is not unique leading to different event definitions. In case of ambiguities the event interpretation which is closest to the nucleon decay hypothesis is chosen.

A minimum distance of $25 \mathrm{~cm}$ is required for the vertex with respect to the surface of the detector. This reduces the fiducial mass to $700 \mathrm{t}$ leading to a sensitivity of $2.0 \mathrm{kt} \cdot \mathrm{y}$.

5.2.3 Data analysis. Topological and kinematical (e.g. single particle momenta, $E, p$, angles, invariant mass(es) etc.) cuts are applied to reduce the neutrino induced background for nucleon decay. Since the misinterpretation probability for the particle type levels to $10-20 \%$ at low energies the accepted topological configurations in the analysis of a specific nucleon decay channel may differ slightly from what is expected based on our knowledge of the decay products.

\subsection{Analysis 3}

5.3.1 Simulation and background. The decay of the nucleon inside the nucleus is treated according to model B. The detector response to the decay particles is simulated in detail using the Monte Carlo program GHEISHA [28]. Electromagnetic showers are described with the EGS code [29] which has been modified in order to deal with low energy electrons and photons.

The neutrino Monte Carlo is used to determine the background for topological configurations with at least 2 showers. We have analysed a sample of events equivalent to a $17.4 \mathrm{kt} \cdot \mathrm{y}$ exposure of the Fréjus detector to atmospheric neutrinos. For other topological configurations we average the Monte Carlo result with the Aachen Padova data.

5.3.2 Measurement of the events. The Fréjus data and the simulated events are scanned and measured on a graphic terminal. A vertex is defined and the hits are associated with tracks and showers. The simulated nucleon decay events are processed channel by channel. A test was made comparing the results to those obtained by mixing some channels and shows that the adopted procedure does not cause a significant scanning bias $[26, \mathrm{c}]$. We restrict our analysis to topological configurations which agree with the expected decay products except allowing for missing showers.

According to the test-detector analysis [30] the probability for pions of momentum lower than $500 \mathrm{MeV}$ to have a secondary interaction with two visible prongs starting from the interaction point is less than $1 \%$. Therefore we reject these events in the nucleon decay analysis.

A contained event covers a volume which must not cross the detector boundaries. Its extension around the vertex is determined by the ends of the tracks and showers (extended by a safety region). The effective mass remaining for nucleon decay vertices is calculated for each simulated decay channel. These masses range from $550 \mathrm{t}$ for $p \rightarrow \mu^{+} \pi^{0}$ to $675 t$ for $p \rightarrow e^{+} \rho^{0}$ depending on the investigated topology and kinematics.

5.3.3 Data analysis with constrained fits. We adopted the method of constrained fits [31] in order to take into account the complete covariance matrix $V$ of each event. Starting from the measured momenta $\mathbf{K}_{\text {meas }}$ we minimize

$\chi^{2}=\left(\mathbf{K}_{\text {meas }}-\mathbf{K}_{\text {fit }}\right) \cdot V^{-1} \cdot\left(\mathbf{K}_{\text {meas }}-\mathbf{K}_{\text {fit }}\right)$

with the constraints to the sum over all particles $\left|\sum p_{i}\right|=p_{F}$ and $\left(\sum E_{i}\right)^{2}=m_{N}^{2}+p_{F}^{2}$. The nucleon mass $m_{N}$ inside the nuclear potential and the Fermi momentum $p_{F}$ are taken from the expectations of nuclear model B. Monte Carlo events are used to parametrize the covariance matrix for each decay channel. Because of the nuclear absorption of pions in the detector the distributions of their reconstructed momenta are strongly non Gaussian. We correct for this by assigning asymmetrical errors to each pion momentum. Additional constraints on the invariant mass of the decay products of mesons are also incorporated.

The probability distribution function* $P\left(v, \chi^{2}\right)$ makes possible a quantitative comparison of different hypotheses for each event. The difference in the shapes of the $\chi^{2}$-distributions of the nucleon decay simulation and the neutrino background provides a comprehensive cut to reduce the background. As an example the $\chi^{2}$-distributions are presented in Fig. 6a for the decay channel $p \rightarrow \mu^{+} K^{0}, K_{s}^{0} \rightarrow \pi^{+} \pi^{-}$and for the neutrino background. The fact that there are events close to the expected energy and momentum but with large $\chi^{2}$ values (Fig. 6b) shows

\footnotetext{
* $v$ is the number of degrees of freedom for the fit which is determined by the number of decaying meson masses plus the number of constraints (four) in energy and momentum minus the unknown direction (two) of the Fermi momentum
} 

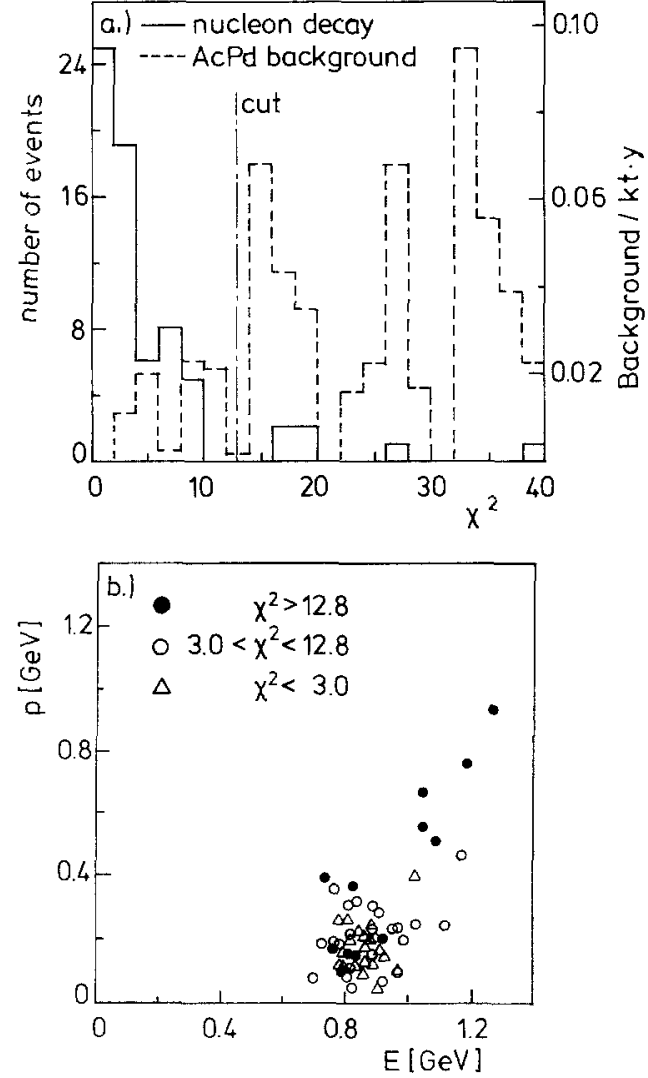

Fig. 6a, b. a shows for the decay mode $p \rightarrow \mu^{+} K^{0}, K_{s}^{0} \rightarrow \pi^{+} \pi^{-}$the $\chi^{2}$-distributions of simulated nucleon decay events and of the background events expected from the Aachen-Padova data. b scatter plot $p$ vs. $E$ for the same nucleon decay events grouped into three $\chi^{2}$-intervals. The smaller dispersion in energy and momentum compared to Fig. 5a reflects the large influence of the nuclear effects on the $\pi^{0}$

Table 3. Summary of results of the three analyses. The "independent" backgrounds and the numbers of candidates have been calculated avoiding double counting of events contributing to more than one decay channel. These numbers are meaningful only within the framework of one specific analysis

\begin{tabular}{lllcl}
\hline $\begin{array}{l}\text { Anal- } \\
\text { ysis }\end{array}$ & $\begin{array}{l}\text { \# analysed } \\
\text { channels }\end{array}$ & \begin{tabular}{l} 
mean backgr. \\
\cline { 4 - 4 } channel $\cdot \mathrm{kt} \cdot \mathrm{y}$
\end{tabular} & $\begin{array}{l}\text { “independent" } \\
\text { backgrounds }\end{array}$ & $\begin{array}{l}\text { Candi- } \\
\text { dates }\end{array}$ \\
\hline 1 & 21 & 0.75 & 4.3 & 2 \\
2 & 20 & 0.3 & 5.3 & 3 \\
3 & 26 & 0.55 & 10.9 & 7 \\
\hline
\end{tabular}

that this method also takes into account the $K^{0}$-mass. Among the 4 events belonging to this topology there are no events inside the chosen cut in the Fréjus data.

\subsection{Comparison of the three analyses}

The details of the three analyses are summarized in Table 2 for two examples. Table 3 shows for each analysis the good agreement between the predicted background and the total number of observed candidates. All these events are complex and difficult to interpret. Among the 12 selected events none is found as a candidate for nucleon decay in all three analyses suggesting background as their origin.

\section{Results}

The $90 \%$ confidence level limit for the lifetime $\tau_{N}$ is calculated according to

$\frac{\tau_{N}}{\mathrm{BR}} \geqq \frac{N_{N}}{S_{0}}$

where $N_{N}$ is the number of protons or neutrons per kiloton in our detector $\left(N_{p}=2.83 \cdot 10^{32} \mathrm{kt}^{-1}\right.$ and $N_{n}=3.20 \cdot 10^{32} \mathrm{kt}^{-1}$ ) and BR is the unknown branching ratio of the investigated nucleon decay channel. The upper limit $S_{0}$ for the signal corrected for efficiency and meson branching fraction is normalized to $1 \mathrm{kt} \cdot \mathrm{y}$ and depends on the confidence level CL required. For $m$ investigated decay modes of the meson produced in a specific nucleon decay channel, CL is evaluated* from [3]:

$\mathrm{CL}=\frac{\int_{0}^{S_{0}} \prod_{i=1}^{m} P\left(N_{C i}, N_{D i}+N_{B i}\right) \cdot \mathrm{d} S}{\int_{0}^{\infty} \prod_{i=1}^{m} P\left(N_{C i}, N_{D i}+N_{B i}\right) \cdot \mathrm{d} S}$

with the Poisson-probabilities:

$$
\begin{aligned}
& P\left(N_{C i}, N_{D i}+N_{B i}\right)=\frac{1}{\mathrm{~N}_{C i} !} \\
& \cdot\left(\varepsilon_{i} \cdot L_{i} \cdot b_{i} \cdot S+N_{B i}\right)^{N_{C i}} \cdot \mathrm{e}^{-\left(\varepsilon_{i} \cdot L_{i} \cdot b_{i} \cdot S+N_{B i}\right)}
\end{aligned}
$$

The number of nucleon decays $N_{D_{i}}$ is given by the product of the efficiency $\varepsilon_{i}$, the sensitivity $L_{i}$, the mesonic branching fraction $b_{i}$ and the corrected signal $S . N_{C i}$ and $N_{B i}$ are the corresponding numbers of candidates and expected background events.

Table 4 gives the results for 29 investigated decay channels. If the limit for a given channel is available in all three analyses we take the middle value. For channels analysed only by two groups the more conservative limit is chosen. The range column shows the lifetime results of the other analysis(es) and gives an indication of the systematic uncertainties.

The behaviour of negative and positive electrons, muons and pions is very similar in our detector. Therefore the limits given are still valid if the charges are reversed (e.g. $\tau_{n \rightarrow e^{+} \pi^{-}} \approx \tau_{n \rightarrow e^{-} \pi^{+}}$).

The nucleon decay channels investigated cover a wide range of event topology in our detector. Other nucleon decay modes not yet analysed in full detail should fall within these topological classes. Since the candidate level found is very low and in addition quite compatible with the background from neutrino events our results imply that possible other channels will also show no signal. In this sense the results presented here are more general than the specific results presented in Table 4.

* The formula used here although different from that presented in [7] gives similar results. The deviations are always smaller than the dispersion between the various analyses 
Table 4. Lower limits on the nucleon lifetime with $90 \%$ confidence level. The analysis presented for a given channel has been chosen according to the procedure described in the text. Corrections for meson decay modes not studied in this analysis are included. (We give $\sum \varepsilon_{i}, b_{i}$ where $b_{i}$ is the meson branching ratio and $\varepsilon_{i}$ the efficiency for the investigated mode.) $N_{B} \leqq 0.1$ means that no background event has been found but depending on the underlying statistics this corresponds to a $66 \%$ confidence level limit on the background. The column $\tau_{N}^{*}$ gives non background subtracted limits. The signature for the decay into $e^{+}\left(\mu^{+}\right) K_{L}^{0}$ is one isolated shower (muon track) and secondaries due to the $K_{L}^{0}$-interaction clearly separated from the shower (the muon track). (The $K_{L}^{0}$-Fe cross section of 1.4 barn corresponds to a mean free path of $30 \mathrm{~cm}$ in the Fréjus detector.) The studied decay modes of the $K^{* 0}, \eta$ and $\omega$ are $K_{S}^{0} \pi^{0} ; 3 \pi^{0}, \pi^{+} \pi^{-} \pi^{0}, \gamma \gamma ;$ and $\pi^{+} \pi^{-} \pi^{0}, \pi^{0} \gamma$ respectively

\begin{tabular}{|c|c|c|c|c|c|c|c|}
\hline Channel & $\sum_{i} \varepsilon_{i}, b_{i}$ & $N_{b}$ & $N_{C}$ & Sens. $(k t \cdot y)$ & $\frac{\tau_{N}^{*}}{\operatorname{BR}\left(10^{31} y\right)}$ & $\frac{\tau_{N}}{\operatorname{BR}\left(10^{3 !} y\right)}$ & $\frac{\tau_{N}}{\operatorname{BR}\left(10^{31} \mathrm{y}\right)}-$ Range \\
\hline$p \rightarrow e^{+} \pi^{0}$ & 0.36 & 0.5 & 0 & 1.58 & 7.0 & 7.0 & $4.4-8.1$ \\
\hline$p \rightarrow e^{+} K_{S}^{0}$ & 0.40 & 0.5 & 0 & 1.56 & 7.6 & 7.6 & $5.6-10.0$ \\
\hline$p \rightarrow e^{+} K_{L}^{0}$ & 0.18 & $\leqq 0.1$ & 0 & 2.00 & 4.4 & 4.4 & - \\
\hline$p \rightarrow e^{+} K^{0}$ & & & & & 6.0 & 6.0 & \\
\hline$p \rightarrow e^{+} \rho^{0}$ & 0.15 & 2.2 & 0 & 1.56 & 2.9 & 2.9 & $2.1-3.2$ \\
\hline$p \rightarrow \mu^{+} \pi^{0}$ & 0.44 & 0.2 & 0 & 1.50 & 8.1 & 8.1 & $4.4-8.2$ \\
\hline$p \rightarrow \mu^{+} \eta^{0}$ & 0.20 & 0.8 & 1 & 1.56 & 2.3 & 2.6 & $2.6-5.3$ \\
\hline$p \rightarrow \mu^{+} K_{S}^{0}$ & 0.34 & 1.2 & 0 & 1.56 & 6.4 & 6.4 & $5.8-9.0$ \\
\hline$p \rightarrow \mu^{+} K_{L}^{0}$ & 0.18 & $\leqq 0.1$ & 0 & 2.00 & 4.4 & 4.4 & - \\
\hline$p \rightarrow \mu^{+} K^{0}$ & & & & & 5.4 & 5.4 & \\
\hline$p \rightarrow u^{+} \rho^{0}$ & 0.05 & 0.5 & 0 & 1.83 & 1.2 & 1.2 & $1.1-2.0$ \\
\hline$n \rightarrow \mu^{+} \rho^{-}$ & 0.10 & 1.1 & 0 & 1.56 & 2.2 & 2.2 & $1.0-2.8$ \\
\hline$p \rightarrow e^{+} e^{+} e^{-}$ & 0.77 & 0.1 & 0 & 1.56 & 14.7 & 14.7 & $13.8-16.9$ \\
\hline$p \rightarrow e^{+} \mu^{+} \mu^{-}$ & 0.33 & 0.16 & 0 & 2.00 & 8.1 & 8.1 & - \\
\hline$p \rightarrow e^{+} \gamma$ & 0.68 & 0.3 & 0 & 1.59 & 13.3 & 13.3 & - \\
\hline$p \rightarrow e^{+} \gamma \gamma$ & 0.76 & 0.8 & 1 & 1.57 & 8.6 & 10.0 & - \\
\hline$p \rightarrow e^{+} \pi^{+} \pi^{-}$ & 0.11 & 2.2 & 0 & 1.56 & 2.1 & 2.1 & $2.1-3.6$ \\
\hline$p \rightarrow e^{+} \pi^{0} \pi^{0}$ & 0.30 & 0.5 & 1 & 1.58 & 3.4 & 3.8 & $3.8-5.6$ \\
\hline$p \rightarrow \mu^{+} \mu^{+} \mu^{-}$ & 0.58 & 0.2 & 0 & 1.67 & 11.9 & 11.9 & $11.1-12.2$ \\
\hline$p \rightarrow \mu^{+} e^{+} e^{-}$ & 0.39 & $\leqq 0.1$ & 0 & 2.00 & 9.1 & 9.1 & - \\
\hline$p \rightarrow u^{+} \gamma$ & 0.84 & 0.1 & 0 & 1.50 & 15.5 & 15.5 & - \\
\hline$p \rightarrow \mu^{+} \pi^{+} \pi^{-}$ & 0.11 & 2.6 & 1 & 1.56 & 1.2 & 1.7 & $1.2-2.9$ \\
\hline$p \rightarrow \mu^{+} \pi^{0} \pi^{0}$ & 0.19 & 0,9 & 1 & 2.00 & 2.8 & 3.3 & - \\
\hline$n \rightarrow e^{+} \pi^{-} \pi^{0}$ & 0.17 & 0.8 & 1 & 2.00 & 2.7 & 3.2 & $2.6-3.5$ \\
\hline
\end{tabular}

\section{Systematic and statistical errors}

As the three analyses use different methods as well as different nuclear models the variation of the results gives an estimate of systematic uncertainties (see Table 4).

For simple topological configurations without any strongly interacting particle (e.g. $p \rightarrow e^{+} e^{+} e^{-}$) there is a variation of $\Delta \tau_{N} / \tau_{N}=15 \%$ among the three different analyses. On the other hand the efficiency for decay channels, with e.g. a $\rho$-meson, is decreased mainly by the influence of the strong interaction inside the parent nucleus. This is model dependent and responsible for variations upt to $60 \%$. The different analysis strategies and the different background calculations also influence the lifetime limits.

Within the framework of a given analysis (Sect. 5.3), the variation of experimental parameters and the choice of nuclear models is used to get information about the specific sources of systematic uncertainties and their influence on the lifetime limits:

A variation of the flash chamber efficiency changes the energy calibration of showers. During the running time of the Frejus experiment the flash chamber efficiency varied at most by $10 \%$. This results in $5 \%$ change of the lifetime limits $\Delta \tau_{N} / \tau_{N}$, reaching at maximum $8 \%$ for $p \rightarrow e^{+} \pi^{0}$.

- The sensitivity to the Geiger tube efficiency depends on the visible energy and the spatial extension for a given decay channel. A $10 \%$ decrease in Geiger efficiency does not affect the lifetime limits for channels with large visible energy (e.g. $p \rightarrow e^{+} \pi^{0}$ ). The maximum change of $\Delta \tau_{N} /$ $\tau_{N}=35 \%$ is obtained for the channel $p \rightarrow \mu^{+} \omega^{0}$ in which the visible energy is close to the trigger threshold.

- Comparing the efficiencies obtained with the nuclear models A and B we estimate that the influence of their 
uncertainty on the lifetime limits is $25 \%$. In the worst case of two poins interacting inside the nucleus the effect reaches $50 \%$.

- The statistical error of the calculation of the recognition efficiency is $\Delta \varepsilon / \varepsilon=0.06 \cdot \sqrt{\frac{1-\varepsilon}{\varepsilon}}$ for 300 generated
events per channel.

- The uncertainty in the background calculation can be estimated by comparing the two methods (cf. Sect. 4). The predictions differ on average by 0.45 event for a sensitivity of $1.7 \mathrm{kt} \cdot \mathrm{y}$. Together with the overall uncertainties on the neutrino flux a predicted background of 1.0 may vary by \pm 0.6 event. However, the influence on the lifetime limits is small. While the background has no effect at all in the case that no candidate has been found the change is $\Delta \tau_{N} / \tau_{N}=8 \%$ in the case of one candidate.

Therefore we conclude that the uncertainties on the obtained lifetime limits level to $\Delta \tau_{N} / \tau_{N}=30 \%$ for most of the investigated channels. For channels dominated by the nuclear effects the uncertainties reach $\Delta \tau_{N} / \tau_{N}=60 \%$.

\section{Conclusions}

We report on the investigation of the nucleon decay into channels with a charged lepton using a kiloton fine grain tracking calorimeter. No evidence is found for nucleon decay and we obtain lower lifetime limits in the range of $10^{31}-1.5 \cdot 10^{32}$ years. The use of different nuclear models and the background calculation with two entirely different methods leads to a reliable estimate of systematic uncertainties.

With another detection technique the two large water Cerenkov experiments, IMB [2] and Kamiokande [3], have reached a higher sensitivity $(4.4$ and $3.8 \mathrm{kt} \cdot \mathrm{y}$ respectively). Since the resolution of the Kamiokande and the Frejus detector is comparable for the decay modes studied in common the Kamiokande experiment obtained in general higher lifetime limits. The large IMB detector especially built for the simple topological classes predicted by SU (5) yields only limits comparable to those presented in this paper for most of the decay modes. The good resolution for multiparticle final states enables us to set new lifetime limits for eight three body decay modes. In the three experiments, the number and nature of the candidate events are compatible with the background expected from atmospheric neutrino interactions.

Improving the present limits with future detectors by one order of magnitude would require not only a substantial increase of the detector sensitivity but also a much better background rejection capability than obtained in present detectors. This would imply more experimental work to determine the neutrino background and to understand precisely the nuclear reinteraction effects.

Acknowledgements. We are very grateful to the technicians whose support in the detector maintenance was essential. We would also like to thank our scanning and measuring teams for their efficient work in processing the data. The interest and support of Prof. H. Faissner is also greatfully acknowledged.

\section{References}

1. P. Langacker: Phys. Rep. 72 (1981) 185

2. S. Seidel et al.: Phys. Rev. Lett. 61 (1988) 2522

3. K.S. Hirata et al.: Phys. Lett. B 220 (1989) 308

4. G. Battistoni et al.: Phys. Lett. B 133 (1983) 454

5. M.R. Krishnaswamy et al.: Nuovo Cimento 9C (1986) 167

6. T.J. Phillips et al.: Phys. Lett. B 224 (1989) 348

7. Ch. Berger et al.: Nucl. Phys. B 313 (1989) 509

8. Ch. Berger et al.: Phys. Lett. B 240 (1990) 237

9. Ch. Berger et al. : Experimental determination of neutrino background expected for a nucleon decay experiment in the Fréjus detector, to be submitted to Nucl. Instrum. and Methods $\mathbf{x x x}$

10. Ch. Berger et al.: Phys. Lett. B 227 (1989) 489

11. Ch. Berger et al.: Nucl. Instrum. and Methods A 262 (1987) 463

12. J. Feltesse et al.: Nucl. Phys. B 93 (1975) 242; H. Samara et al.: Nucl. Phys. B 161 (1979) 1; D.M. Binnie et al.: Phys. Rev. D8 (1973) 2789

13. G. Höhler: $\pi N$-scattering, Landhold-Börnstein, numerical data and functional relationship in science and technology. New Series, Vol. I/9b1 and I/9b2. H. Schopper (ed.). Berlin, Heidelberg, New York: Springer 1982, 1983

14. R. Hofstadter: Ann .Rev. Nucl. Sci. 7 (1957) 231

15. J. Hüfner, H. Thies: Phys. Rev. C 20 (1979) 273

16. E. Oset et al.: Nucl. Phys. A 448 (1986) 597

17. G.E. Brown et al.: Phys. Lett. 118 B (1982) 39

18. J. Bystricky et al. : Scattering of elementary particles, LandholdBörnstein, numerical data and functional relationship in science and technology. New Series, Vol. I/9. H. Schopper (ed.). Berlin, Heidelberg, New York: Springer 1982, 1983

19. C. Mahaux: The interaction between medium energy nucleons in nuclei. 1982, AIP Conference Proceedings No. 97. H.O. Meyer (ed.). New York: AIP 1983

20. J. W. Negele: Commun. Nucl. Part. Phys 12 (1983) 1

21. I. Navon et al.: Phys. Rev. Lett. 42 (1979) $1465 ;$ K. Nakai et al.: Phys. Rev. Lett. 44 (1980) 1446; D. Ashery et al.: Phys. Rev. C 23 (1981) 2173; S.M. Levenson et al.: Phys. Rev. C 28 (1983) 326

22. G. Barr, T.K., Gaisser, T. Stanev: Phys. Rev. D 39 (1989) 3532

23. E.V. Bugaev, V.A. Naumov: Phys. Lett. 232 B (1989) 391

24. M. Honda et al.: Phys. Lett. B 248 (1990) 193

25. H. Faissner et al.: Phys. Lett. B 68 (1977) 377

26. a) J.-F. Glicenstein: thesis 1989, DPhPE-Saclay France;

b) H.J. Daum: Habilitationsschrift 1990, WUB90-6, BUGH Wuppertal;

c) H. Mönch: thesis 1990, PiTHA 91/02, RWTH Aachen

27. R. Brun et al.: CERN, DD, EE, 84-1 (1987)

28. H. Fesefeldt: The simulation of hadronic showers, RWTH Aachen PITHA 85/02 (1985)

29. R.L. Ford, W.R. Nelson: SLAC Report 210 (1978)

30. B. Voigtländer: thesis 1985; RWTH Aachen

31. A.G. Frodesen, O. Skjeggestad, H. Tøfte: Probability and statistics in particle physics, Universitetsforlaget Oslo 1979 